\title{
Exposing latent fingermarks on problematic metal surfaces using Time of Flight Secondary lon Mass Spectroscopy
}

\author{
Tshaiya Devi Thandauthapani ${ }^{1}$, Adam J. Reeve ${ }^{1}$, Adam S. Long ${ }^{2}$, Ian J. Turner ${ }^{3}$ \& James S. Sharp ${ }^{1, *}$ \\ ${ }^{1}$ School of Physics and Astronomy, University of Nottingham, Nottingham, NG7 2RD, UK. \\ ${ }^{2}$ College of Life and Natural Science, University of Derby, Kedleston Road, Derby, DE22 1GB, UK. \\ ${ }^{3}$ Centre for Excellence in Learning and Teaching, University of Derby, Kedleston Road, Derby, \\ DE22 1GB, UK.
}

*Corresponding author email: james.sharp@nottingham.ac.uk;

\begin{abstract}
Fingermarks are a key form of physical evidence for identifying persons of interest and linking them to the scene of a crime. Visualising latent (hidden) fingermarks can be difficult and the correct choice of techniques is essential to develop and preserve any fingermarks or other (e.g. DNA) evidence that might be present. Metal surfaces (stainless steel in particular) have proven to be challenging substrates from which to reliably obtain fingermarks. This is a great cause for concern among police forces around the globe as many of the firearms, knives and other metal weapons used in violent crime are potentially valuable sources of fingermark evidence. In this study, a highly sensitive and non-destructive surface science technique called time of flight secondary ion mass spectroscopy (ToF-SIMS) was used to image fingermarks on metal surfaces. This technique was compared to a conventional superglue based fuming technique that was accompanied by a series of contrast enhancing dyes (basic yellow 40 (BY40), crystal violet $(\mathrm{CV})$ and sudan black (SB)) on three different metal surfaces. The conventional techniques showed little to no evidence of fingermarks being present on the metal surfaces after a few days. However, ToF-SIMS revealed fingermarks on the same and similar substrates with an exceptional level of detail. The ToF-SIMS images demonstrated clear ridge definition as well as detail about sweat pore position and shape. All structures were found to persist for over 26 days after deposition when the samples were stored under ambient conditions.
\end{abstract}




\section{Introduction}

Violent crimes involving firearms and knives are on the rise in the UK and are at their highest levels since 2011 [1]. Identification of the perpetrators of these crimes is a key priority for both UK and global law enforcement agencies. The development of new methodologies for quick and reliable identification of trace evidence is therefore vital if these agencies are to keep pace with increasing crime rates.

Fingermarks have traditionally provided valuable physical evidence for the identification (or elimination) of persons of interest in criminal investigations. The pattern of friction ridges that form on the surface of the underside of the palm, fingers, and thumbs are believed to be unique to each individual and therefore provide a valuable means of identification [2]. Fingermarks have been used for over 100 years and have been successful in securing evidence used in most of the convictions [3]. A major source of fingermark evidence comes from what is typically referred to as latent fingermarks. These marks are often deposited by individuals when they directly interact with a surface, or handle an object, resulting in the transfer of sweat and other constituents from the hand and fingers onto the object. Latent fingermarks are not normally visible to the naked eye and require a development process that makes them observable.

A typical latent fingermark constitutes either eccrine secretions or a combination of eccrine and sebaceous secretions from human sweat [4]. The eccrine sweat glands are found all over the surface of the skin and secrete around $98 \%$ water with dissolved salts, amino acids, urea, proteins and a small amount of lipids [5]. Sebaceous sweat glands are also found all over the body (except the palms of the hands and soles of the feet) and are typically localised to regions containing hair follicles. These glands secrete an oily fluid called sebum which is composed of triglycerides $(\sim 41 \%)$, wax esters $(\sim 26 \%)$, squalene $(\sim 12 \%)$, and free fatty acids $(\sim 16 \%)[4,6]$.

Over the past century, a multitude of techniques and reagents have been developed for enhancing the visualisation of latent fingermarks containing both eccrine and sebaceous secretions [2]. These include, but are not limited to, ninhydrin [7], powder development [8], vacuum metal deposition (VMD) [9], physical developers [10], small particle reagents [11], and cyanoacrylate (CNA, superglue) fuming [12]. The choice of technique or reagent being used in each case has been optimised to account for the composition of the fingermarks and the physical and chemical properties of the surface/substrate on which the fingermark is believed to have been deposited [4]. Factors such as the porosity and wettability of the substrate $[4,13]$ are key in choosing the appropriate technique for developing the fingermarks, as is the potential for damage that could be caused by some of the more destructive development techniques (e.g. VMD). 
One important class of substrates that have proven difficult to develop fingermarks on are simple metals and alloys. The most popular method that has been used to attempt to retrieve latent fingermarks from these surfaces has been based on conventional enhancement technique which is cyanoacrylate fuming [14]. Additional contrast enhancement techniques such as fluorescent dyes (e.g. basic yellow 40 (BY40)) [15], vacuum metal deposition [16], powder dusting [17] (non-magnetic and magnetic powders), gun blue [15, 18], acidified hydrogen peroxide [19, 20], crystal violet (CV) [21], and sudan black (SB) [22] have also been employed. These additional contrast enhancement techniques can give fingermark examiners a better opportunity of visualising a latent fingermark. However, many of them have limited success in enhancing latent fingermarks on metal surfaces [23]. This is due to a number of factors involving the environmental conditions that the metal surfaces were exposed to at the crime scene, the age of the fingermark that has been deposited, the nature of the interactions between the fingermark residue and the metal surfaces, and the way in which any developing reagents may react with the surface [4].

One extreme example where numerous attempts have been made to retrieve fingermarks from metallic alloy surfaces is in the examination of fired brass bullet casings [24, 25]. The high temperatures, pressures, and mechanical friction that bullet casings were exposed to during firing often results in the obliteration of any fingermarks that may have been present - so much so that even the most sensitive surface science techniques cannot detect any residual traces of material. Stainless steel knife blades and guns also have the potential to provide valuable sources of fingermark evidence and to link persons of interest to the scene of crime. However, methods that reliably allow forensics science to develop and enhance fingermarks on these surfaces have proven to be elusive [16].

The suboptimal performance of conventional fingermark visualisation techniques has driven the development of new applications of existing technologies. For example, processes that involve the deposition of electrochromic polymers onto metal surfaces have been used to coat regions of metal surfaces between fingermark ridges and generate negative images of the fingermark residue [26-28]. $\mathrm{Xu}$ et al. [29] have also proposed a technique for the visual enhancement of sebaceous latent fingermarks by electrochemiluminescence (ECL) which gave a satisfactory visual contrast. In addition to this, scanning electrochemical microscopy (SECM) [30], scanning Kelvin microprobe [31, 32] and electrostatic deposition $[24,25]$ techniques have also been reported to visualise identifiable latent fingermarks on metal surfaces.

Time-of-flight secondary ion mass spectroscopy (ToF-SIMS) is a technique that has been used to successfully image latent fingermarks. This surface sensitive technique uses an ion beam, which is rastered across a sample and used to ablate material on the surface with sub-monolayer precision. Secondary ions are emitted during the ablation process that are specific to the individual molecular species present on the surface. Ions with different mass-to-charge ratios are detected and can be used to 
provide a spatial map of the presence of trace amounts of deposits such as those found in finger ridge secretions. ToF-SIMS has been used by Hinder and Watts to obtain clear images of fingermarks on organic substrates such as silicon wafers, carrier bags, cling-film, photocopier paper, poly (vinylidene difluoride) PVdF coating, polyester coating, and newspaper [33]. Szynkowska et al. have also confirmed that SIMS can be used to produce images of complete fingermarks on inorganic surfaces such as metals and metal alloys. However, the images produced in the study by Szynkowska and coworkers were deemed to be of poor quality [34]. Bailey et al. managed to obtain better images of fingermarks deposited on hand grenades made of galvanised steel substrate after periods of up to 48 hours following deposition [21].

The study presented here demonstrates that ToF-SIMS can be used to visualise latent fingermarks on stainless steel and other metal surfaces when conventional cyanoacrylate and staining techniques have been shown to yield no results. The imaging technique is shown to be able to visualise latent fingermarks on these surfaces for a range of donors of different gender, age, and ethnicity. Moreover, the images obtained using this technique were shown to be of sufficient quality to display detailed information about the pattern of ridges with pore level detail across the entire fingermark for periods of up to 26 days after deposition.

\section{Materials \& Methods}

Disks of brass, aluminium, and stainless steel approximately $30 \mathrm{~mm}$ in diameter and $1 \mathrm{~mm}$ thick were cleaned prior to fingermark deposition by placing them in a beaker of methanol and sonicating for 15 minutes. A further 15 minutes of sonication was then performed in a beaker of distilled, deionised water to remove water soluble/polar contaminants from the surfaces. No evidence of fingermarks or other contaminants were observed on the metal surfaces following these cleaning steps.

\begin{tabular}{|c|c|c|c|}
\hline Donor & Gender & Ethnicity & Age \\
\hline A & Male & White British & 20 \\
\hline B & Female & Asian (Indian) & 27 \\
\hline C & Male & Asian (Indian) & 28 \\
\hline D & Male & White British & 43 \\
\hline E & Male & White British & 44 \\
\hline F & Female & White British & 49 \\
\hline
\end{tabular}

Table 1: Details of the donors that participated in the fingerprint deposition experiments.

Fingermarks were deposited on separate disks of each metal by six different donors (see Table 1) using a protocol based on UK Home Office internal guidelines [3, 35]. Briefly, hands were washed thoroughly with soap and water to remove excess sebaceous material and any contaminants on the skin. 
Donors were then made to wear powder-free nitrile gloves (VWR) for 30 minutes to allow predominantly eccrine secretions to accumulate on the fingertips. Fingermarks were deposited using the right index finger which was pressed down onto the surface of metal disks and then lifted off. The maximum force applied during deposition was maintained at a constant $2 \mathrm{~N}$ for two seconds in all experiments and was measured by placing the disks on a weighing scale (Model CS 5000E, Ohaus Corporation) during the deposition process. In each case the same finger was used to deposit six sequential marks on to different disks of the same type of metal. This was done in order to form a depletion series to determine how repeated contact influenced the quantity and morphology of fingermark secretions that were deposited on each of the metal substrates studied. After fingermark deposition, the disks were attached to microscope glass slides using double sided adhesive tabs (Leit Adhesive Carbon Tabs 12mm, Agar Scientific) and kept in a closed microscope slide box in room at a temperature of $22+/-2{ }^{\circ} \mathrm{C}$ and $40+/-1 \%$ relative humidity, until required.

A pilot study was performed using one male (Donor A) and one female (Donor B) donor who each deposited a depletion series of six, latent fingermarks on aluminium, brass, and stainless steel disks, every day for a period of 14 days. Fingermarks were deposited following the simple hand cleansing procedure and after wearing gloves for a period of 30 minutes as mentioned above. The sweat that accumulated on the fingertips during this time was used to represent the natural eccrine compounds that are found in a fingermark. After the deposition of each depletion series, samples were stored in a microscope slide box, ready for further treatment and analysis. At the end of the 14 day period, all of the samples were removed and treated simultaneously so that the effects of aging of the fingermarks on each of the substrates could be determined.

The resulting fingermarks were developed using techniques that are employed by East Midlands Special Operations Unit- Forensic Sciences (EMSOU-FS) and other police forensics laboratories across the UK when trying to develop fingermarks on non-porous metal substrates. The techniques were applied in the sequence: cyanoacrylate (superglue) fuming, followed by sequential staining of the cyanoacrylate deposits with the dyes, basic yellow 40 (BY40), crystal violet (CV), and sudan black (SB).

In each case, cyanoacrylate fuming was performed at the EMSOU-FS forensics lab using a superglue fuming cabinet (MVC5000 Mason Vactron, Foster and Freeman). The relative humidity in the fuming chamber was set and maintained at $75-80 \%$ throughout the fuming cycle. Once this humidity had been established, $3.5 \mathrm{~g}$ cyanoacrylate (superglue) was heated to $120^{\circ} \mathrm{C}$ and allowed to form a vapour inside the fuming cabinet chamber. Samples were exposed to the cyanoacrylate vapour for a total of 15 minutes, the cabinet was then purged to remove all traces of the vapour and halt the fuming process. All the samples were fumed simultaneously in the same chamber to ensure that all were exposed to identical fuming conditions. 
BY40 was applied to the fumed metal disks by laying them flat and soaking in the dye $(0.2 \%$ $\mathrm{w} / \mathrm{v}$ in ethanol) for five seconds before rinsing in water and drying in a stream of air at room temperature . The disks were then imaged using a Nikon D7200 digital camera with a yellow filter $(75 \mathrm{~mm} \times 75 \mathrm{~mm}$ No.12, Kodak Wratten gelatin filter, excitation wavelength: $510-530 \mathrm{~nm}$ ) under blue light illumination with CrimeLite ${ }^{\circledR} 2$ light source emitting wavelengths in the range $420-470 \mathrm{~nm}$ (Foster and Freeman). The samples were then immersed for five minutes each in CV $(0.03 \% \mathrm{w} / \mathrm{v}$ in $1: 50$ ethanol/water mixture) and then SB (1\% w/v in 2:1 ethanol/water mixture) dyes. After each successive dying process, the metal substrates were rinsed with water and dried in a stream of air before being imaged using the same camera under white light illumination (Crimelite ${ }^{\circledR}$ 2, Foster and Freeman, wavelength range 400 $-700 \mathrm{~nm}$ ) without any filters. In all cases, illumination and imaging conditions were kept constant when collecting images from samples using each setup described above.

Images of the fingermarks that were developed using the cyanoacrylate staining techniques were then examined and graded according to the scheme presented in Table 2. Grading of the fingermarks was performed by the same fingerprint officer and in the same sitting using images rather than using the live samples. In determining the exact score for each fingermark, a holistic approach was adopted, whereby each image was viewed as a whole against each descriptor. The degree of clarity evidenced by minimal distortion was prioritised over the amount of ridge detail present in determining the most appropriate score. This overall approach was deemed appropriate for allowing consistency in the grading across all of the samples studied.

In the present study, fingermarks from within a given depletion series were found to be very similar in appearance on each of the substrates studied and for each of the staining techniques used. This similarity of the marks obtained from each deposition in a given depletion series allowed for quantitative information about the quality of fingermarks to be obtained for each day in the pilot aging study. 


\begin{tabular}{|c|c|}
\hline Score & Definition \\
\hline 1 & No visible ridges or contacted area - Nothing of interest \\
\hline 2 & $\begin{array}{c}\text { Weak development; evidence of contacted area but no visible ridge } \\
\text { details present }\end{array}$ \\
\hline 3 & $\begin{array}{c}\text { Partial development; up to 1/3 of ridges present including clear evidence } \\
\text { of ridge flow and pattern (first level detail) and partial evidence of } \\
\text { individual ridge minutiae - bifurcations and ridge endings (second level } \\
\text { detail). Significant distortion evident. }\end{array}$ \\
\hline 4 & $\begin{array}{c}\text { Strong development; between 1/3 and 2/3 of ridges present including } \\
\text { clear evidence of ridge flow and pattern (first level detail), individual } \\
\text { ridge minutiae - bifurcations and ridge endings (second level detail) and } \\
\text { partial evidence of pore position and shape (third level detail). Minor } \\
\text { distortion evident }\end{array}$ \\
\hline 5 & $\begin{array}{c}\text { Very strong development; }>2 / 3 \text { of ridges present including clear } \\
\text { evidence of ridge flow and pattern (first level detail), individual ridge } \\
\text { minutiae - bifurcations and ridge endings (second level detail) and pore } \\
\text { position and shape (third level detail). Very minor or no obvious } \\
\text { distortion evident. }\end{array}$ \\
\hline
\end{tabular}

Table 2: Scoring scheme used to describe the quality of fingermarks developed using cyanoacrylate fuming followed by BY4O, CV, and SB dyes for each of the three metal surfaces studied (aluminium, brass, and stainless steel) as adapted from Sears et al. [3]. The definitions of first, second and third level of details on the fingermark have been adapted from Jain et al. [36].

The main study involved each of the six different donors (see Table 1) depositing a depletion series of six, predominantly eccrine latent fingermarks on stainless steel disks. Following fingermark deposition, the disks were attached to glass microscope slides using double sided adhesive and stored in a closed microscope slide box $\left(22+/-2^{\circ} \mathrm{C}\right.$ and relative humidity of $\left.40+/-1 \%\right)$. After a total of thirteen days in storage, the fingermarks obtained from each of the six donors were imaged using a ToF-SIMS IV instrument (ION-TOF GmbH., Münster, Germany). In each case, the second deposition in the depletion series was used for imaging (rather than the first) to eliminate the effects of any possible transients that could be present in the first deposition e.g. potential excess of fingermark secretions, initial errors in deposition by participants.

During ToF-SIMS imaging a $25 \mathrm{kV} \mathrm{Bi}_{3}{ }^{+}$primary ion beam delivering a pulsed target current of approximately $1.0 \mathrm{~A}$ was rastered at an angle of $45^{\circ}$ across the surface of the stainless steel disks. The accelerating potential used in the mass spectrometer was $2 \mathrm{keV}$ and the pressure in the imaging chamber was maintained between $1 \times 10^{-7}$ and $1 \times 10^{-8}$ mbar. All samples were placed into the ToF-SIMS chamber at the same time. The samples were imaged at a temperature of $23^{\circ} \mathrm{C}$ and each took approximately 15 minutes to image over an area of $5 \mathrm{~mm} \times 5 \mathrm{~mm}$. A spatial resolution of $0.02 \mathrm{~mm} / \mathrm{pixel}$ and an average of 20 scans were used to obtain each image. The steel sample imaged for Donor A on day thirteen was also reimaged on day twenty six. This second image of the sample was taken over a 
larger area $(18 \mathrm{~mm} \times 18 \mathrm{~mm}$, spatial resolution of $0.01 \mathrm{~mm} /$ pixel, imaging time 150 minutes $)$ of the surface and was acquired using the same imaging parameters described above. Data acquisition and analysis were performed using SurfaceLab 6 software (IONTOF GmbH).

The other five stainless steel samples in the depletion series were kept as control experiments to determine whether there was any loss of material in the fingermark residue after imaging with ToFSIMS (either as a result of the reduced pressure in the imaging chamber or as a result of ablation by the primary ion beam). On day twenty eight, all thirty six samples (including the six samples that went through ToF-SIMS analysis) were fumed together using cyanoacrylate, before being stained with the fluorescent dyes BY40, CV, and SB using identical procedures to those that were used in the pilot study.

\section{Results \& Discussion}

Figure 1 shows examples of fingermarks that were developed using cyanoacrylate fuming followed by staining of the cyanoacrylate deposits with the dyes BY40, CV, and SB. Examples of images are shown for fingermarks that were developed on each of the metal disks after periods of three and eight days respectively following deposition. Each of the samples was inspected immediately after cyanoacrylate fuming and no evidence of fingermarks could be detected. In this sense, each sample could be rated with a score of 1 according to the scheme proposed in Table 2 . This inability to detect fingermarks on metal surfaces using cyanoacrylate fuming alone is common and promotes the need for further staining of the cyanoacrylate deposits with dyes that are capable of improving the optical contrast of any latent fingermarks that may be present. There was, however, clear evidence of fingermarks following BY40 staining of the cyanoacrylate on both aluminium and brass surfaces after three days. However, the quality of the marks appears degraded after only eight days on both of these surfaces. In the case of stainless steel surfaces, the marks developed using BY40 were found to be unrecognisable as fingermarks after only three days. BY40 fluorescence has previously been shown to be effective for enhancing the contrast and details of eccrine fingermarks on non-porous surfaces following cyanoacrylate fuming $[13,37]$. However, these initial experiments demonstrate that this staining technique gives unreliable results when developing predominantly eccrine fingermarks on metal surfaces such as those studied here. 
Day 3

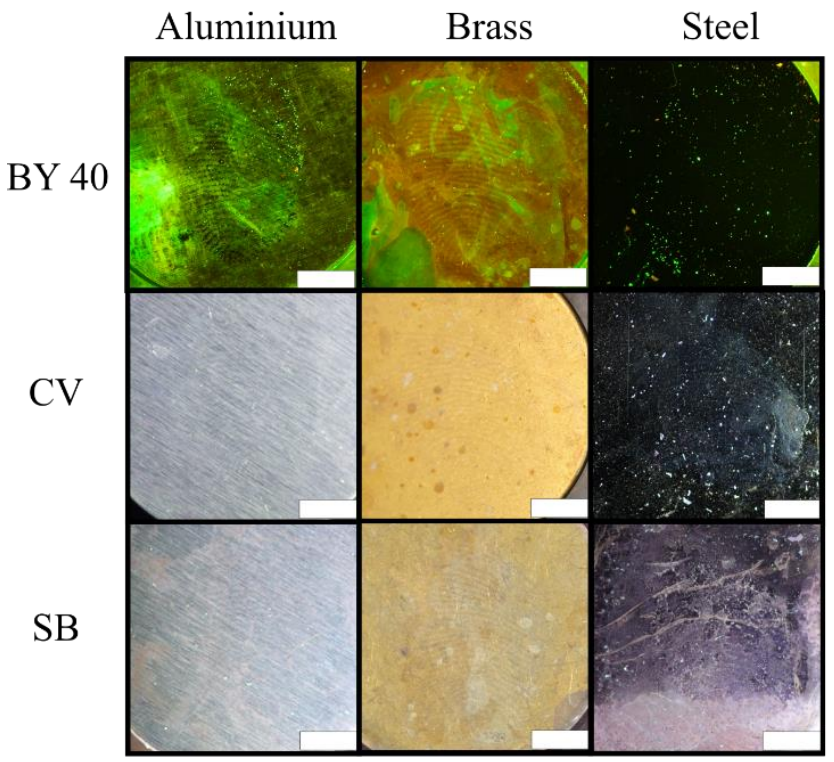

Day 8

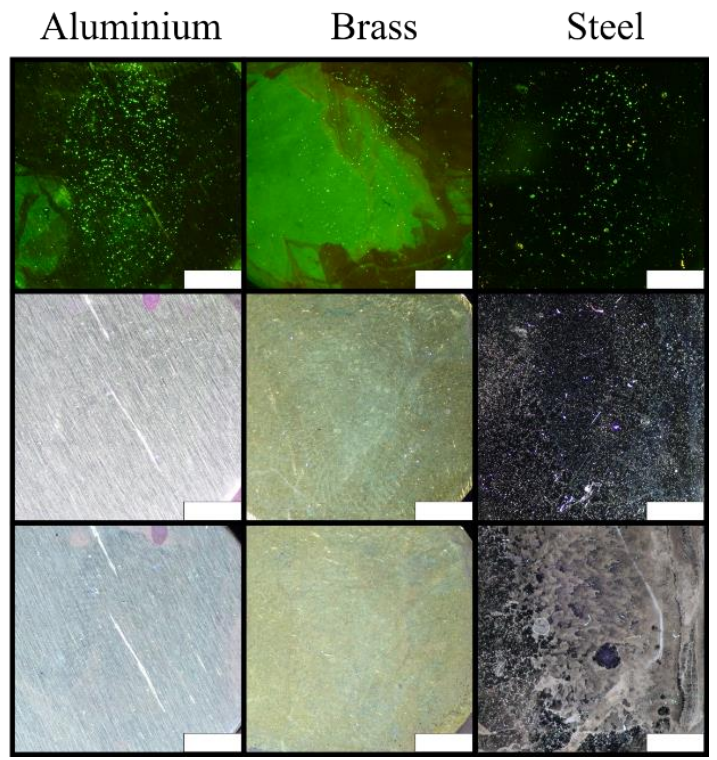

Figure 1: Optical micrographs of representative examples of fingermarks that were developed using cyanoacrylate fuming followed by staining with the dyes BY4O, CV, and SB on each of the three different metals. The left hand panel shows fingermarks that were deposited by Donor A and then developed three days after deposition. The right hand panel shows fingermarks from the same donor that were developed eight days after deposition. The white scale bar corresponds to $5 \mathrm{~mm}$ in all cases.

The second row of images in Figure 1 shows that $\mathrm{CV}$ staining of the cyanoacrylate deposits was capable of developing fingermark ridges after three days of exposure to ambient conditions on the brass substrates. However, this was not the case for aluminium and steel as these substrates varied between having no visible ridges and giving weak development; resulting in mean scores of $1.5 \pm 0.29$ and $1.3 \pm 0.25$ respectively. The third row of images shows that enhancement was also possible with SB on all three substrates after only 3 days. The marks observed on brass were generally found to be of a higher quality than those found on aluminium substrates, which were in turn better than steel substrates. Although there was some residual evidence of fingermarks on brass and aluminium after three days, little to no evidence of fingermarks was observed on all three of the metals studied after eight days. It was observed that marks were generally less prevalent when developed with SB and CV than with BY40. This is to be expected because these dyes are used to detect sebaceous deposits and the fingermarks used here contained predominantly eccrine material. Crystal violet (CV) binds to lipids (fats) and to dead skin cells in the fingermark residue while sudan black (SB) preferentially dissolves into lipids that are present in larger quantities in sebaceous secretions and further enhances any contrast obtained by prior CV staining [37]. We stress that these two dyes were used in order to reproduce 
standard operating protocols in UK forensic laboratories and the fact that they do not stain for the eccrine components that are targeted in the present study perhaps makes their use unreasonable for the present work. However, the occasional observation of fingermarks following the use of these two stains does not rule out their use entirely. The fact that some features were observed suggest that the dyes are capable of detecting skin cells or residual sebaceous deposits on the surface of the metal substrates which may not have necessarily been observed using cyanoacrylate fuming alone.

In each case, digital images of samples that were stained with each of the dyes were collected, compiled and graded/scored according to the scheme presented in Table 2. Plots of the mean scores of six images obtained from the depletion series performed on each metal surface are given in Figure 2 as a function of the number of days following deposition. In each case, the error bars were obtained from the standard error of the scores given to each mark in the depletion series. As mentioned above, the similarity in the appearance of marks in a given depletion series allowed for quantitative information about the quality of the fingermarks to be obtained for each day in the aging experiments. 

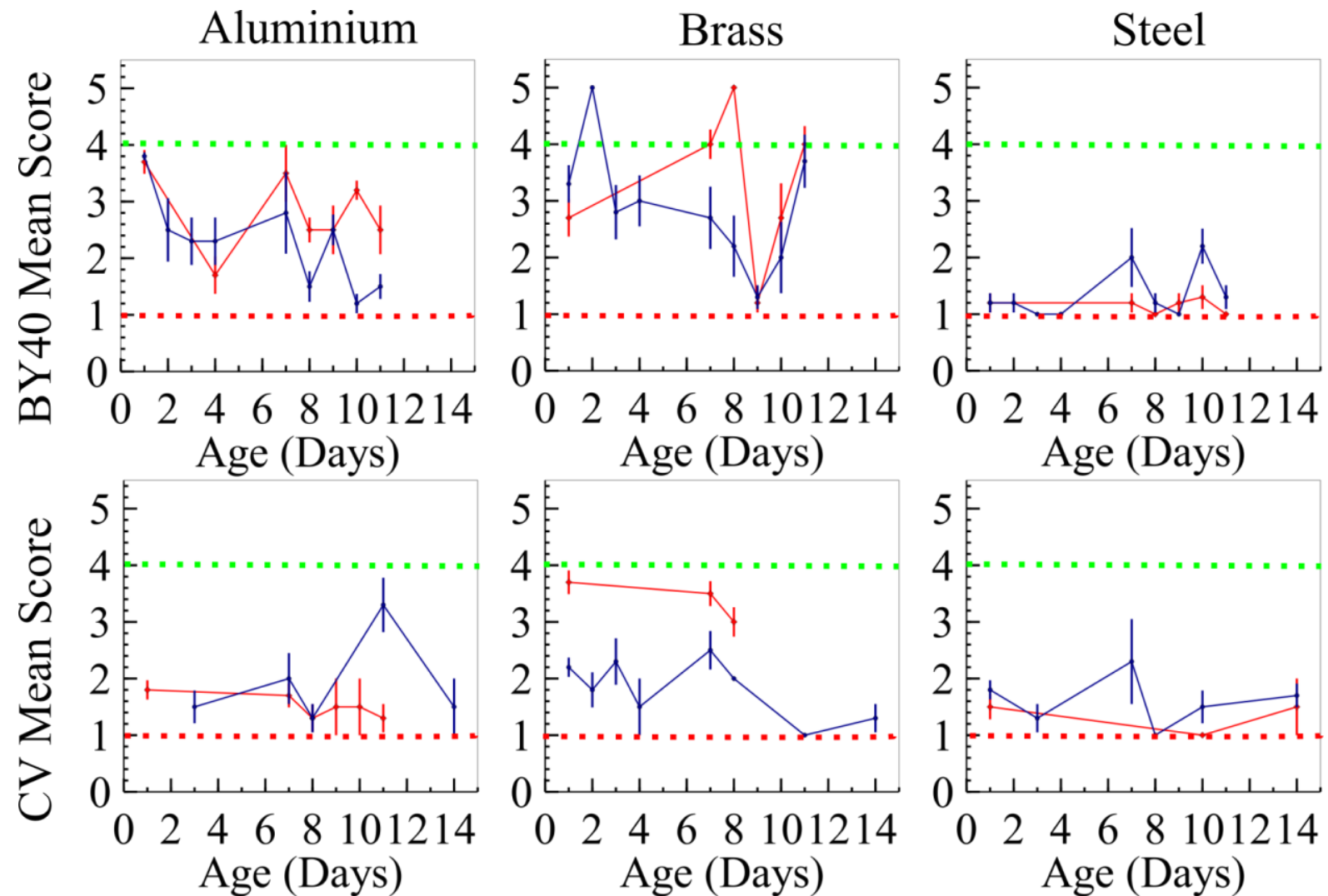

Age (Days)

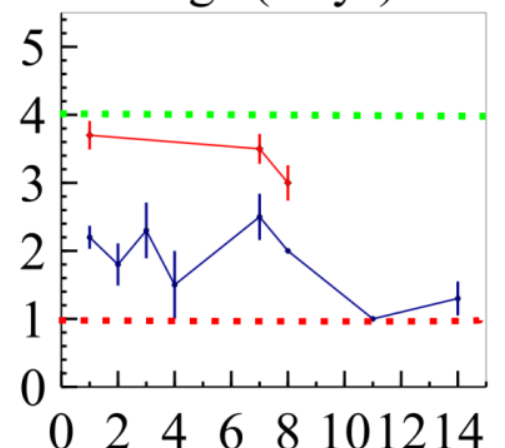

Age (Days)

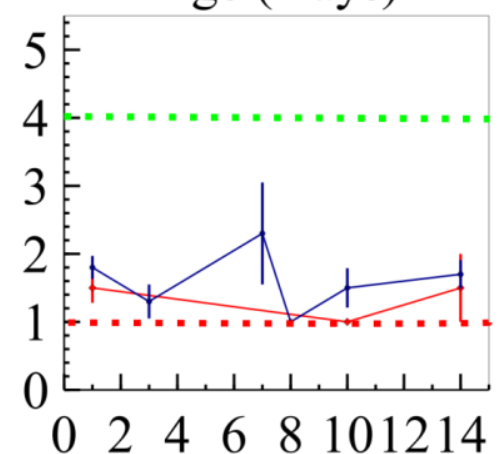

Age (Days)
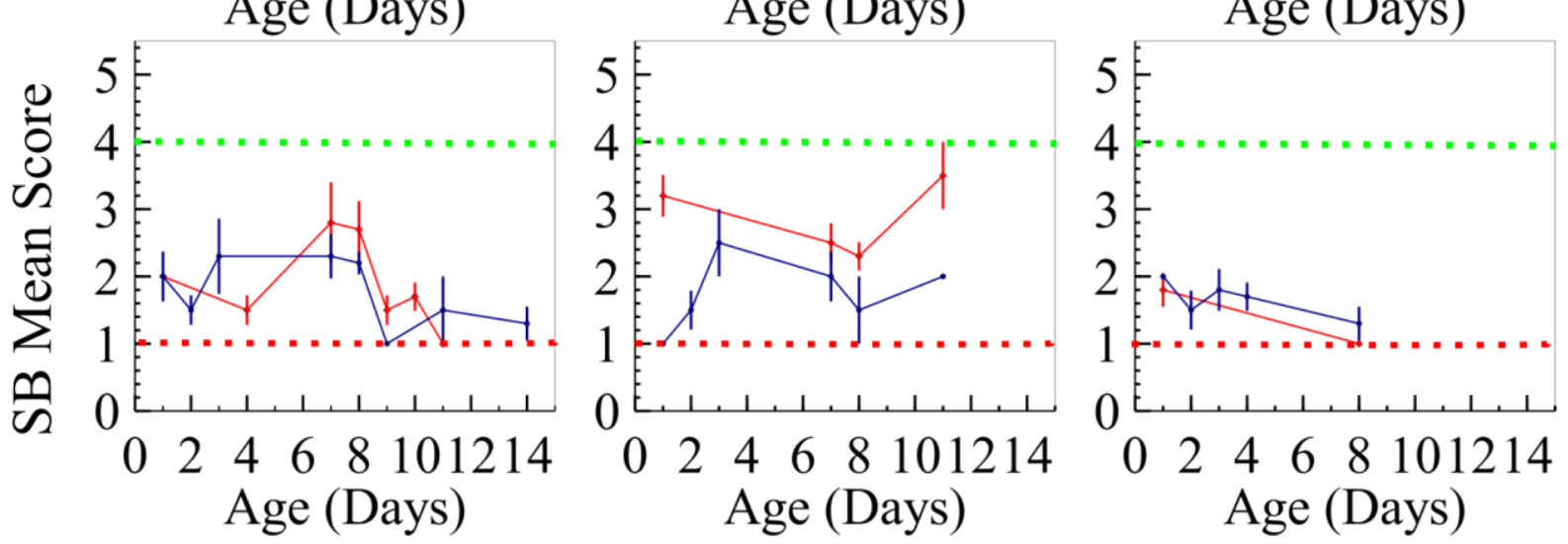

Figure 2: Mean scores for two different donors (Donors A and B) as a function of time after deposition for the three different staining techniques. The blue line corresponds to the mean score for the fingermarks deposited by Donor A while the red line represents mean score of fingermarks deposited by Donor B. Error bars were calculated using the standard error of the mean of scores obtained from fingermarks in the same depletion series $(n=6)$. The horizontal red dotted line denotes the score for which no traces of a fingermark can be detected (score $\leq 1$, see Table 2) while the green dotted horizontal line represents the threshold score above which a fingermark is considered to be acceptable for identification purposes (score $\geq 4$ ).

Figure 2 shows that BY40 staining initially gives clear evidence of ridge flows of fingermark providing acceptable and identifiable marks (mean score between 3 and 4) on both aluminium and brass but the quality of the marks steadily decreases with aging time. In the case of SB and CV staining, the quality of the marks was observed to be significantly lower than for BY40 in the case of brass and aluminium (as expected based on the predominantly eccrine nature of the deposits). 
In the case of the steel surfaces, very few fingermarks were able to be recovered using any of the staining protocols even after just one day following deposition (mean score 1-2). No visible ridges were observed on these surfaces. In fact, steel consistently displayed significantly poorer quality fingermarks compared to the other two metal surfaces over the duration of the 14 day experiment. This is clearly a cause for concern for forensic practitioners trying to visualise fingermarks on knife blades and firearms. As steel reproducibly failed to provide identifiable fingermarks for all the staining techniques, this metal substrate was chosen as the focus for more detailed studies using ToF-SIMS.

ToF-SIMS has the capability to obtain high spatial resolution images of the chemical properties of the sample via the acquisition of highly localised mass spectra of material deposited on a surface. Acquiring mass spectra at each position on the sample surface enables the construction of images of the distribution of particular chemical species on a substrate. In particular, these images are constructed by plotting the number of counts obtained for secondary ions with a given mass-to-charge ratio as a function of position on the surface. As different materials emit different types and quantities of secondary ions when bombarded with the primary ion beam, the spatial variation in the type and quantity of emitted secondary ions can be used to build up highly specific chemical maps of any deposited material. The difference in the chemical properties of the substrate and any thin layer of fingermark residue deposited on it can therefore, be detected with a high sensitivity (femtomolar) and high spatial resolution $(\sim 0.002 \mu \mathrm{m})[38]$.

A number of different positive and negative secondary ionic species were identified in the regions where fingermarks had been deposited by each of the six donors. Images of the spatial distribution of these ions on the substrate surface are given in Figures 3 and 4. The images demonstrate excellent ridge detail including first and second level detail (See Table 2 for definitions), as well as clearly disclosed third level detail in the form of the position and localisation of individual ridge pores and unique individual ridge profiles/ridge edges. These observations are true for all the donors irrespective of gender, age, and ethnicity. There is also a clear contrast between the fingermark and the substrate on steel samples equivalent to those which showed no evidence of fingermarks when stained and imaged using conventional techniques. According to the scoring system described in Table 2, the quality of the ridges shown in the ToF-SIMS images corresponds to a score of 5 (exceptional). Despite the fact that these fingermarks were imaged 13 days after deposition, they still exhibit high level details with individual epidermal ridge patterns and sweat pores.

The greatest contrast in the images came from $\mathrm{K}^{+}$and $\mathrm{Na}^{+}$(Figure 3) which are among the most common ions found in fingermark residue. $\mathrm{Ca}^{2+}$ ions were also present on the surface, albeit at lower concentrations $[6,39]$. The presence of a $\mathrm{C}_{3} \mathrm{H}_{3}{ }^{+}$ion was also detected at higher concentrations on the steel substrate than in the fingermark regions, although its' origin is not clear. De Hoffmann [40] and Goacher et al. [41] detected a similar ion in ToF-SIMS studies of lignin and polysaccharides, but noted 
that this ion falls within the mass resolution of the $\mathrm{K}^{+}$ion. Assignment of the $\mathrm{C}_{3} \mathrm{H}_{3}{ }^{+}$ion could therefore be erroneous. An additional organic ion $\left(\mathrm{C}_{3} \mathrm{H}_{8} \mathrm{~N}^{+}\right)$was also present in the fingermark regions as shown in Figure 3. This ion is most likely due to the presence of the dimethyl (methylene) ammonium ion - a fragment from precursor amino acids such as serine or the betaines (choline or glycine betaine) [42].

In the negative ionic species that are shown in Figure 4, the $\mathrm{Cl}^{-}$ion was the least prevalent. The presence of this ion could be expected given that this is the most abundant negative ion found in fingermark residue. However, the images in Figure 4 show that the substrate regions in between the fingermark contain more $\mathrm{Cl}^{-}$than the fingermark regions. This negative contrast suggests that the chloride ions were present on the surface and were removed by the contacting finger. Excess $\mathrm{Cl}^{-}$ions on the surface could originate from the washing procedure that was used to clean the metal surfaces i.e. residual salt traces dissolved in the water.

Sulphite ions $\left(\mathrm{SO}_{3}{ }^{2-}\right)$ produced the greatest positive contrast among the negative ions for all of the donors studied. Again, the reasons for the presence of sulphite ions is not entirely clear, but sulphites are one of the most widely used food preservatives in the UK [43]. Sulphate $\left(\mathrm{SO}_{4}{ }^{2-}\right)$ ions were also present in the fingermark residues obtained from all the donors. These metabolites can be found in high concentrations in food such as bread, nuts, alcohol (fermented beverages), dried fruits, and vegetables [44]. Dihydrogen phosphate $\left(\mathrm{H}_{2} \mathrm{PO}_{4}^{-}\right)$ions were also detected in the fingermark residue. The presence of these ions is most likely due to the presence of trace amounts of phospholipid in the fingermarks [45]. An additional high molecular weight ion $\left(\mathrm{C}_{12} \mathrm{H}_{25} \mathrm{SO}_{4}{ }^{-}\right)$, was also observed. This ion was observed by Poleunis et al. [46] and was identified in the residue from household washing treatments and during treatments involving personal care products containing sulphur surfactants such as dodecyl benzene sulphonic acid [47]. Hence, this ion is likely to originate from the hand washing procedure used in these experiments.

In addition to the data shown in Figure 3 and Figure 4, a number of additional positive and negative ions were identified (not shown). These includes positive secondary ion fragments of lipids [48] which correspond to free fatty acids such as dodecanoic acid, tridecanoic acid, tetradecanoic acid, palmitic acid, stearic acid, and cholesterols with mass (m/z 201, 215, 229, 257, 285, 369 and 387). However, the signal intensities were much weaker in comparison to the strong signals obtained from the inorganic $\mathrm{Na}^{+}$and $\mathrm{K}^{+}$ions present in the predominantly eccrine fingermark residues (Figure 3 ). The negative ion spectra of the fingermarks also contained evidence of additional secondary ions that are consistent with the presence of lipids such as the phosphite ion $\left(\mathrm{PO}_{3}^{-}\right)$observed at $\mathrm{m} / \mathrm{z} 78.96$ [45]. 


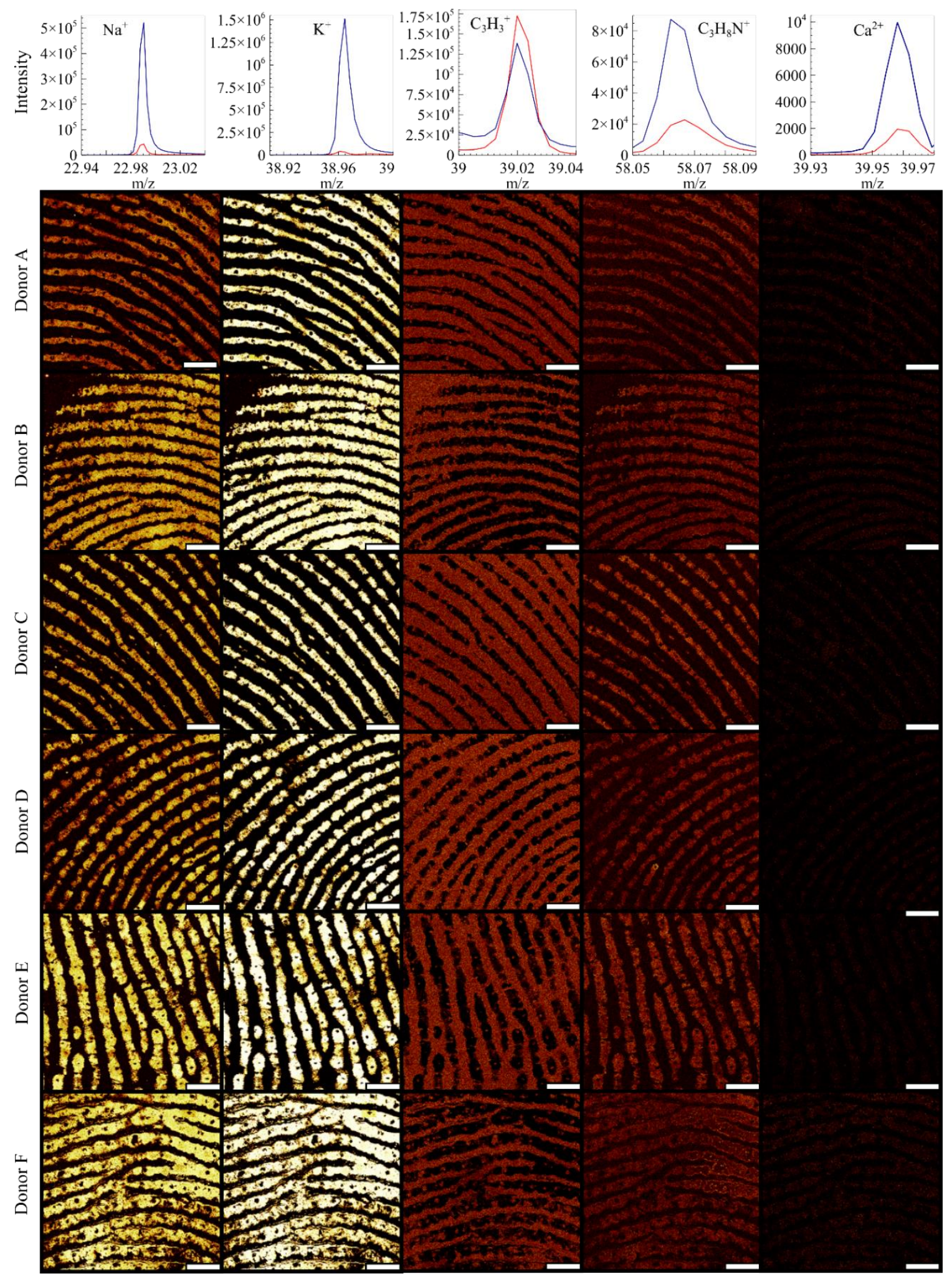

Figure 3: ToF-SIMS imaging of fingermarks on stainless steel surfaces after 13 days of deposition. The top row shows typical ToF-SIMS spectra corresponding to Donor B for the positive secondary ions that were emitted from the surface of the samples. In each case, the ionic species responsible for the peak is labelled. The blue line represents a typical spectrum taken from a region on the fingermark and the red line represents the spectrum collected from the substrate. The images below each spectrum show spatial variations in the number of counts/signal intensity measured at each position on the sample surface. Data are shown for each of the six donors. Each image took approximately 15 minutes to acquire. Scale bar: $1 \mathrm{~mm}$. 

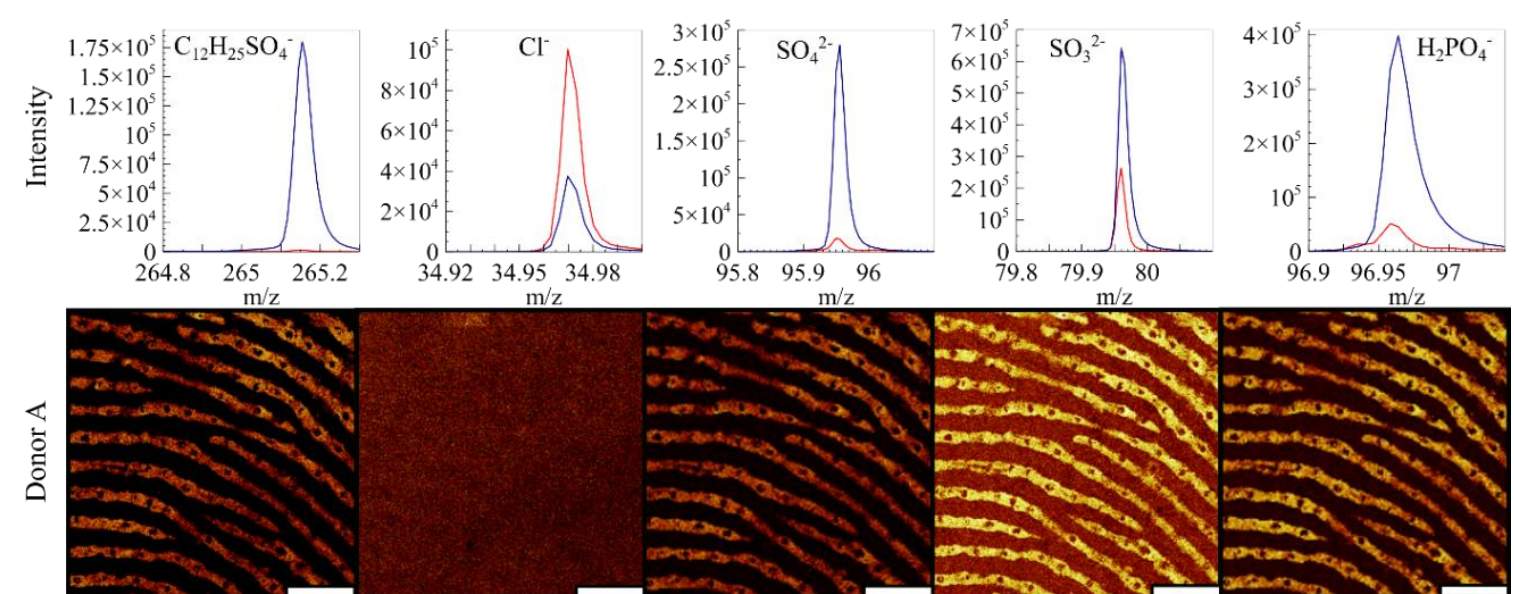

๓๐
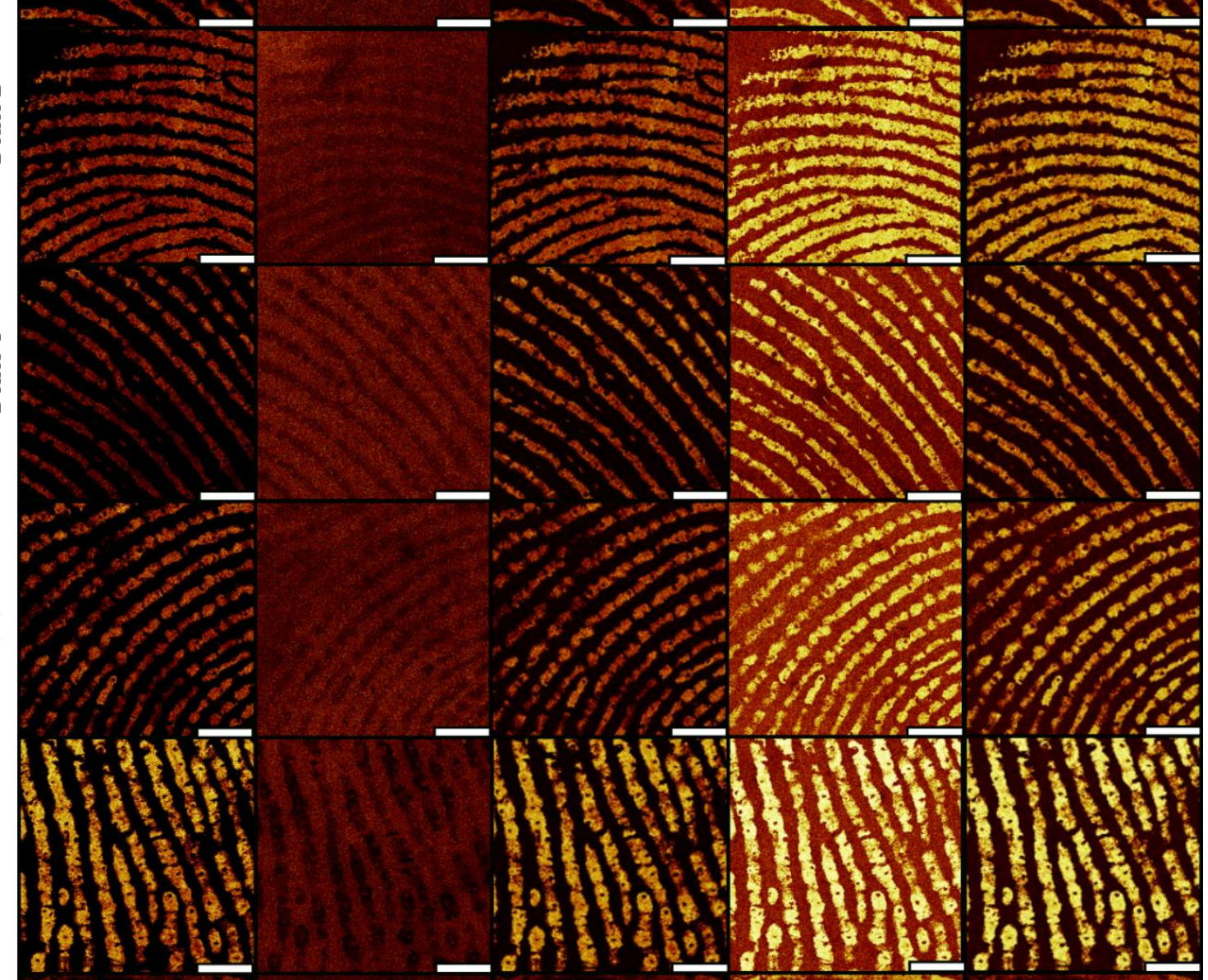

\section{西}
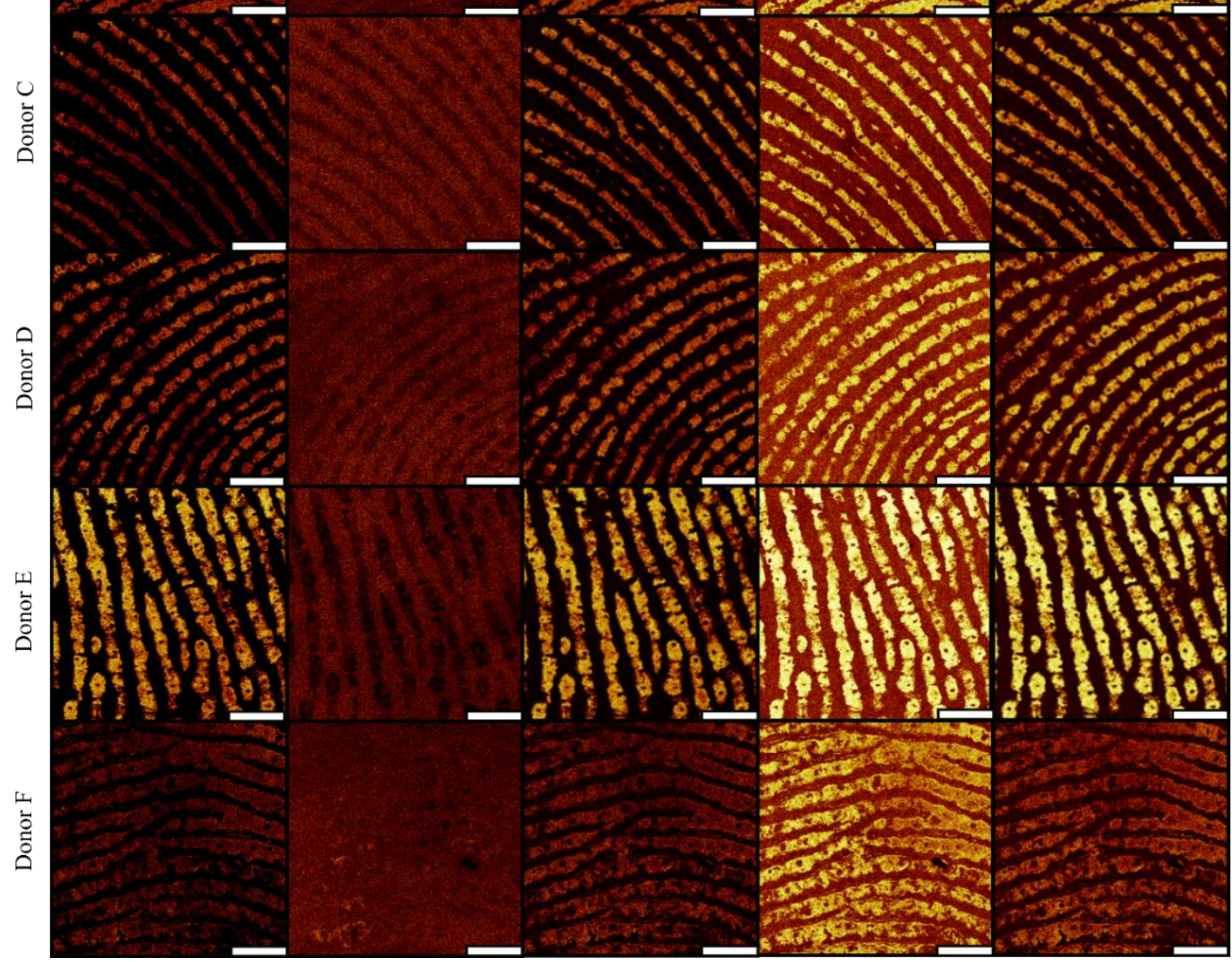

Figure 4: ToF-SIMS imaging of fingermarks on stainless steel surfaces after 13 days of deposition. The top row shows typical ToF-SIMS spectra of Donor E for the negative secondary ions that were emitted from the surface of the samples. In each case, the ionic species responsible for the peak is labelled. The blue line represents a typical spectrum taken from a region on the fingermark region and the red line represents the spectrum collected from the substrate. The images below each spectrum show spatial variations in the number of counts/signal intensity measured at each position on the sample surface. Data are shown for each of the six donors. Each image took approximately 15 minutes to acquire. Scale bar: $1 \mathrm{~mm}$. 
Figure 5 shows a large area $(18 \mathrm{~mm} \times 18 \mathrm{~mm})$ scan of the $\mathrm{K}^{+}$ion distribution in a fingermark that was deposited on stainless steel by Donor A and imaged using ToF-SIMS after a period of twenty six days following deposition. Remarkably, this image exhibits a similar level of detail to the images shown in Figures 3 and 4 despite the fact that more conventional techniques (BY40, CV, and SB) show no evidence of useable marks after this period of time. The insets shown in Figure 5 are photographs of the same sample that was removed from the ToF-SIMS chamber, fumed using CNA and stained using the three dyes, BY40, CV, and SB according to the protocol described previously. These images show the same region of the sample that was imaged using ToF-SIMS and display no evidence of the fingermark shown in the main panel. One potential criticism of this approach is that exposure to the high vacuum conditions in the ToF-SIMS chamber could have removed much of the material in the fingermark and thus rendered the stains useless once the sample had been imaged using ToF-SIMS. However, samples which had been aged for the same time but without being placed in the ToF-SIMS chamber were also stained using the same dyes and showed no evidence of fingermarks when 
photographed. In fact, the digital photograph images of the stained samples that were not exposed to high vacuum conditions were very similar to those shown in figures $5 b$ ), c) and d).

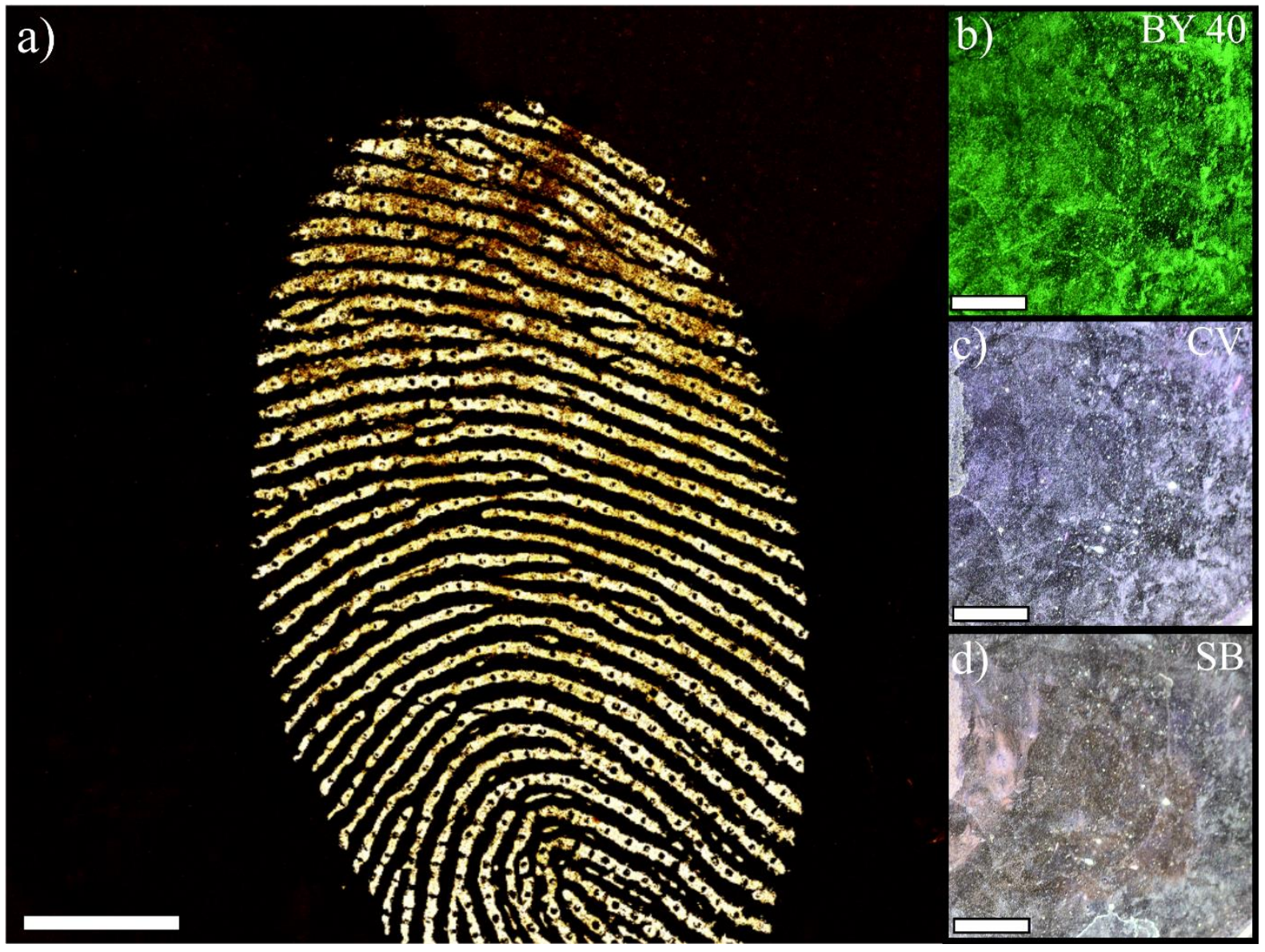

Figure 5: ToF-SIMS image of an entire fingermark of stainless steel a) Image obtained from Donor A, 26 days after deposition. The image shows the spatial distribution of the $K+$ ion. This fingermark is the same as that shown in Figure 3, after a further 13 days of aging. Collection of this high res image took 150 minutes. The scale bar corresponds to $3 \mathrm{~mm}$. The same sample shown in a) was treated with b) $B Y 40$, c) $C V$ and d) SB was captured using conventional photographic techniques. All three images/photographs have the same scale bar of $5 \mathrm{~mm}$ and depict the same region of the same fingermark shown in a).

It is clear from the data shown in Figure 5 that ToF-SIMS can be used to visualise an entire fingermark with an exceptional level of detail (first, second, and third level detail being evident) on a steel surface despite it being aged for twenty six days. Whereas, when the same steel sample was treated with BY40, $\mathrm{CV}$, and SB the procedure described in Table 2 consistently gave a score of one as no fingermark evidence could be observed on the surfaces. Residual traces of components such as potassium $\left(\mathrm{K}^{+}\right)$and sodium $(\mathrm{Na}+)$ ions as well as a number of less abundant ions remain on the surface even after exposure to the high vacuum imaging conditions used in ToF-SIMS. Notably, the sample imaged in Figure 5 was 
also one of the samples that was imaged after thirteen days (see Figures 3 and 4). This demonstrates that the ToF-SIMS imaging technique is non-destructive and can be repeatedly used to image fingermarks without compromising their quality. The application of ToF-SIMS therefore has the potential far reaching implications and applications in the detection of latent fingermarks and could be used to more reliably link persons of interest to the scene of a crime.

This study clearly demonstrates that fingermarks that have been deposited on metal (stainless steel) surfaces can be visualised with an exceptional level of detail using ToF-SIMS. The resulting images are significantly better than those obtained using conventional development techniques on the same/similar samples (which show no visible marks). However, further studies on 'real-world' samples are required to demonstrate the applicability of ToF-SIMS to a broader range of fingermarks and samples surfaces. The fingermarks used in this study were produced to contain consistent levels of eccrine and sebaceous secretions. However, real-world samples are likely to contain variable amounts of these deposits. Such variability could influence the level of detail recovered using ToF SIMS as well as potentially altering the degradation times of associated with fingermarks that are present on the samples. The steel surfaces that were used in this work also had a flat surface topology and a relatively small size (30mm diameter). Real forensic samples are likely to have greater roughness and/or to have curved surfaces. Moreover, such samples are likely to be quite large and one potential limitation of the work performed here is that the maximum sample size that can be imaged using the ToF-SIMS instrument is $9 \mathrm{~cm} \times 9 \mathrm{~cm}$. While this is acceptable for many objects, it may not be large enough for a gun or a knife and some cutting of the samples may be required.

ToF-SIMS also has the potential to be used to image fingermarks following the use of other imaging techniques. However, the efficacy of the technique under these circumstances is likely to depend upon the nature of the previous imaging technique that was used (destructive or non-destructive) and on the chemical properties of any contrast agents that may have been used to enhance the fingermark. If a contrast agent was used to try to enhance a mark and subsequently covered it up, then the initial ToF SIMS signal would be dominated by the secondary ions that are emitted from the contrast agent rather than the fingermark. However, repeated rastering of the primary ion beam could be used to etch away at the any coating layers to reveal the fingermark as has been done previously for polymer layers [49]. Previous work by Sisco and coworkers [48] has also shown that mass spectroscopy techniques are relatively insensitive to the prior use of contrast agents such as black fingerprint power, magnetic powders and some fluorescent dyes. 


\section{Conclusion}

ToF-SIMS has demonstrated the capability to obtain exceptional quality fingermarks where conventional development techniques produce no evidence of marks. The application of this technique to steel surfaces, in particular, has shown that it is capable of identifying pore level detail on all ridge patterns by imaging the spatial distribution of inorganic ions such as sodium and potassium on metal surfaces. Moreover, samples that have been aged under ambient conditions for up to twenty six days display the same exceptional quality of detail and can be repeatedly imaged with no obvious evidence of deterioration in the quality of the fingermarks produced. ToF-SIMS imaging therefore has the potential and could be a valuable tool in helping to visualise fingermarks on objects such as stainless steel knife blades and firearms.

\section{Acknowledgements}

The authors would like to thank East Midlands Special Operations Unit - Forensic Science (EMSOU-FS) for providing access to the fuming chamber, to Amelia Harrison (University of Derby) for help in organising the dyes and fluorescent crime lights used for the conventional staining techniques and Long Jiang for the help in using the ToF-SIMS instrument.

\section{Funding}

This work was supported by the University of Derby and the UK Home Office.

\section{Author Contributions}

TDT and AJR collected and analysed the data. ASL, IJT and JSS supervised the project and helped to write the manuscript.

\section{References}

1. Flatley, J. Statistical bulletin: Crime in England and Wales: year ending June 2017, Office for National Statistics [GB]: https://www.ons.gov.uk/peoplepopulationandcommunity/crimeandjustice/bulletins/crim einenglandandwales/june2017

2. Lee, H. C., Ramotowski, R., \& Gaensslen, R. E. (Eds.). (2001). Advances in fingerprint technology. CRC press. 
3. Sears, V. G., Bleay, S. M., Bandey, H. L., \& Bowman, V. J. (2012). A methodology for finger mark research. Science \& Justice, 52(3), 145-160.

4. Houck, M. M. (Ed.). (2016). Forensic Fingerprints. Academic Press.

5. Ramotowski, R. (Ed.). (2012). Lee and Gaensslen's advances in fingerprint technology. CRC Press, 339.

6. Girod, A., Ramotowski, R., \& Weyermann, C. (2012). Composition of fingermark residue: a qualitative and quantitative review. Forensic Science International, 223(1), $10-24$

7. Odén, S., \& Von Hofsten, B. (1954). Detection of fingerprints by the ninhydrin reaction. Nature, 173(4401), 449-450.

8. Sodhi, G. S., \& Kaur, J. (2001). Powder method for detecting latent fingerprints: a review. Forensic Science International, 120(3), 172-176.

9. Jones, B. J., Downham, R., \& Sears, V. G. (2012). Nanoscale Analysis of the Interaction between Cyanoacrylate and Vacuum Metal Deposition in the Development of Latent Fingermarks on Low-Density Polyethylene. Journal of Forensic Sciences, 57(1), 196200.

10. Wilson, J. D., Cantu, A. A., Antonopoulos, G., \& Surrency, M. J. (2007). Examination of the steps leading up to the physical developer process for developing fingerprints. Journal of Forensic Sciences, 52(2), 320-329.

11. Jasuja, O. P., Singh, G. D., \& Sodhi, G. S. (2008). Small particle reagents: development of fluorescent variants. Science \& Justice, 48(3), 141-145.

12. Wargacki, S. P., Lewis, L. A., \& Dadmun, M. D. (2007). Understanding the chemistry of the development of latent fingerprints by superglue fuming. Journal of Forensic Sciences, 52(5), 1057-1062.

13. Yamashita, B., \& French, M. (2011). Latent print development. The Fingerprint Sourcebook, 7-67.

14. Girelli, C. M., Lobo, B. J., Cunha, A. G., Freitas, J. C., \& Emmerich, F. G. (2015). Comparison of practical techniques to develop latent fingermarks on fired and unfired cartridge cases. Forensic Science International, 250, 17-26.

15. Dominick, A. J., \& Laing, K. (2011). A comparison of six fingerprint enhancement techniques for the recovery of latent fingerprints from unfired cartridge cases. Journal of Forensic Identification, 61(2), 155.

16. Ramos, A. S., \& Vieira, M. T. (2012). An efficient strategy to detect latent fingermarks on metallic surfaces. Forensic Science International, 217(1), 196-203.

17. Freeman, H. N. (1999). Magnetic fingerprint powder on firearms and metal cartridges. Journal of Forensic Identification, 49(5), 479.

18. Dove, A. (2017). Fingerprint Development on Cartridge Cases Through the Electrodeposition of Gun Blue. Journal of Forensic Identification, 67(3), 391.

19. Edmiston, K. E., \& Johnson, J. (2009). Determining an optimal sequence for chemical development of latent prints on cartridge casings and shotgun shells. Journal of Forensic Sciences, 54(6), 1327-1331.

20. Cantu, A. A., Leben, D. A., Ramotowski, R., Kopera, J., \& Simms, J. R. (1998). Use of acidified hydrogen peroxide to remove excess gun blue from gun blue-treated cartridge cases and to develop latent prints on untreated cartridge cases. Journal of Forensic Science, 43(2), 294-298.

21. Bailey, M. J., Ismail, M., Bleay, S., Bright, N., Elad, M. L., Cohen, Y., Geller, B., Everson, D., Costa, C., Webb, R.P., \& Watts, J. F. (2013). Enhanced imaging of developed fingerprints using mass spectrometry imaging. Analyst, 138(21), 6246-6250.

22. Tilstone, W. J., Savage, K. A., \& Clark, L. A. (2006). Forensic science: An encyclopedia of history, methods, and techniques. ABC-CLIO. 
23. Wightman, G., Emery, F., Austin, C., Andersson, I., Harcus, L., Arju, G., \& Steven, C. (2015). The interaction of fingermark deposits on metal surfaces and potential ways for visualisation. Forensic Science International, 249, 241-254.

24. Bond, J. W., \& Heidel, C. (2009). Visualization of latent fingerprint corrosion on a discharged brass shell casing. Journal of Forensic Sciences, 54(4), 892-894.

25. Bond, J. W. (2008). Visualization of latent fingerprint corrosion of metallic surfaces. Journal of Forensic Sciences, 53(4), 812-822.

26. Bersellini, C., Garofano, L., Giannetto, M., Lusardi, F., \& Mori, G. (2001). Development of latent fingerprints on metallic surfaces using electropolymerization processes. Journal of Forensic Science, 46(4), 871-877.

27. Beresford, A. L., \& Hillman, A. R. (2009). Electrochromic enhancement of latent fingerprints on stainless steel surfaces. Analytical Chemistry, 82(2), 483-486.

28. Brown, R. M., \& Hillman, A. R. (2012). Electrochromic enhancement of latent fingerprints by poly (3, 4-ethylenedioxythiophene). Physical Chemistry Chemical Physics, 14(24), 8653-8661.

29. Xu, L., Li, Y., He, Y., \& Su, B. (2013). Non-destructive enhancement of latent fingerprints on stainless steel surfaces by electrochemiluminescence. Analyst, 138(8), 2357-2362.

30. Qin, G., Zhang, M., Zhang, T., Zhang, Y., McIntosh, M., Li, X., \& Zhang, X. (2012). Label-Free Electrochemical Imaging of Latent Fingerprints on Metal Surfaces. Electroanalysis, 24(5), 1027-1032.

31. Williams, G., McMurray, H. N., \& Worsley, D. A. (2001). Latent fingerprint detection using a scanning Kelvin microprobe. Journal of Forensic Science, 46(5), 1085-1092.

32. Williams, G., \& McMurray, H. N. (2007). Latent fingermark visualisation using a scanning Kelvin probe. Forensic Science International, 167(2-3), 102-109.

33. Hinder, S. J., \& Watts, J. F. (2010). SIMS fingerprint analysis on organic substrates. Surface and Interface Analysis, 42(6-7), 826-829.

34. Szynkowska, M. I., Czerski, K., Grams, J., Paryjczak, T., \& Parczewski, A. (2007). Preliminary studies using imaging mass spectrometry TOF-SIMS in detection and analysis of fingerprints. The Imaging Science Journal, 55(3), 180-187.

35. Bailey, M. J., Bright, N. J., Croxton, R. S., Francese, S., Ferguson, L. S., Hinder, S., Jickells, S., Jones, B.J., Jones, B.N., Kazarian, S.G. \& Ojeda, J. J. (2012). Chemical characterization of latent fingerprints by matrix-assisted laser desorption ionization, time-of-flight secondary ion mass spectrometry, mega electron volt secondary mass spectrometry, gas chromatography/mass spectrometry, X-ray photoelectron spectroscopy, and attenuated total reflection Fourier transform infrared spectroscopic imaging: an intercomparison. Analytical Chemistry, 84(20), 8514-8523.

36. Jain, A. K., Chen, Y., \& Demirkus, M. (2007). Pores and ridges: High-resolution fingerprint matching using level 3 features. IEEE Transactions on Pattern Analysis and Machine Intelligence, 29(1), 15-27.

37. Bleay, S. M., Sears, V. G., Bandey, H. L., Gibson, A. P., Bowman, V. J., Downham, R., Fitzgerald, L., Ciuksza, T., Ramadani, J. \& Selway, C. (2012). Finger mark development techniques within scope of ISO 17025. Fingerprint Source Book, Home Office Centre for Applied Science and Technology (CAST), 233-289.

38. Michel, R., \& Castner, D. G. (2006). Advances in time-of-flight secondary ion mass spectrometry analysis of protein films. Surface and Interface Analysis, 38(11), 13861392.

39. Hirokawa, T., Okamoto, H., Gosyo, Y., Tsuda, T., \& Timerbaev, A. R. (2007). Simultaneous monitoring of inorganic cations, amines and amino acids in human sweat by capillary electrophoresis. Analytica Chimica Acta, 581(1), 83-88. 
40. De Hoffmann, E., \& Stroobant, V. (2007). Mass spectrometry: principles and applications. John Wiley \& Sons.

41. Goacher, R. E., Jeremic, D., \& Master, E. R. (2010). Expanding the library of secondary ions that distinguish lignin and polysaccharides in time-of-flight secondary ion mass spectrometry analysis of wood. Analytical Chemistry, 83(3), 804-812.

42. Patel, V. B., Mehta, K., Munoz-Clares, R. A., Melissas, V. S., Garrow, T. A., Howell, E. E., Crego, A., Curtis, M., Tatke, P.A., Servillo, L. \& Swierczynski, J. (2015). Betaine: Chemistry, Analysis, Function and Effects. Royal Society of Chemistry.

43. Tytgat, G., Bartelsman, J. F. W. M., \& van Deventer, S. J. H. (Eds.). (1995). Inflammatory Bowel Diseases (No. 85). Springer Science \& Business Media.

44. Florin, T., Neale, G., Gibson, G. R., Christl, S. U., \& Cummings, J. H. (1991). Metabolism of dietary sulphate: absorption and excretion in humans. Gut, 32(7), 766773.

45. Heim, C., Sjövall, P., Lausmaa, J., Leefmann, T., \& Thiel, V. (2009). Spectral characterisation of eight glycerolipids and their detection in natural samples using timeof-flight secondary ion mass spectrometry. Rapid Communications in Mass Spectrometry, 23(17), 2741-2753.

46. Poleunis, C., Everaert, E. P., Delcorte, A., \& Bertrand, P. (2006). Characterisation of human hair surfaces by means of static ToF-SIMS: A comparison between $\mathrm{Ga}+$ and $\mathrm{C}$ 60+ primary ions. Applied Surface Science, 252(19), 6761-6764.

47. Committee for Veterinary Medicinal Products, Linear Alkyl Benzene Sulfonic Acids, summary report (1), European Medicines Agency, EMEA/MRL/755/00-FINAL July 2000.

48. Sisco, E., Demoranville, L. T., \& Gillen, G. (2013). Evaluation of C 60 secondary ion mass spectrometry for the chemical analysis and imaging of fingerprints. Forensic Science International, 231(1), 263-269.

49. Bailey, J.B., Havelund, R., Shard, A.G., Gilmore, I.S., Alexander, M.R., Sharp, J.S. \& Scurr, D.J. (2015), 3D ToF-SIMS Imaging of Polymer Multilayer Films Using Argon Cluster Sputter Depth Profiling, ACS Appl. Mater. Interfaces, 7(4), 2654-2659 